\title{
A REUSABLE LEXICAL DATABASE TOOL FOR MACHINE 'TRANSLATION
}

\author{
BRIGITIF BL $\check{A S E R}$ \\ IBM Germany \\ Institute for Knowledgc \\ Based Systems \\ I'.O. Box 103068 \\ W-6900 Heidelberg \\ Email: alschwee \\ at dludibm 1 bitnet
}

\author{
ULRIKF; SCHWALL \\ II3M Germany \\ Institute for Knowledge \\ Based Systems \\ I'.O. Box 103068 \\ W-6900 J Leidelberg \\ schwall \\ at dhdibm l.bitnet
}

\author{
ANGLLIKA STORRER \\ University of 'Tübingen \\ Serninar für \\ natürlichsprachl. Systeme \\ Wilhelmstr. 113 \\ W-7400 Tübingen \\ storrer at arbuckle.sns. \\ neuphilologie.uni-tuebingen.de
}

\section{ABSTRACT}

This paper describes the lexical database tool LOI_A (I.inguistic-Oriented Iexical database Approach) which has been developed for the construction and maintenance of lexicons for the machine translation system L.MT, liirst, the requirements such a tool should meet are discussed, then I.M'I' and the lexical information it requires, and some issues concerning vocabulary acquisition are presented. Afterwards the architecture and the components of the I.OI A system are described and it is shown how we tried to mect the requirements worked out earlier. Although I,OI A originally has been designed and implemented for the German-English I,MT prototype, it aimed from the beginning at a representation of lexical data that can be reused for other LMT or MI' prototypes or even other NLI' applications. $\Lambda$ special point of discussion will therefore be the adaptability of the tool and its components as well as the reusability of the lexical data stored in the database for the lexicon development for $\mathrm{L} \mathrm{MT}^{\prime}$ or for other applications.

\section{Introduction}

The availability of large-scale lexical information has widely been recognized as a bottleneck in the construction of Natural Language Processing (NLP) systems. The lexical database I,OLA has been developed in connection with the Logicprogramming-based Machine 'Translation (LMT') system and shall be presented here. This work is part of the objectives of the project Transicexis launched in 1991 at the Institute of Knowledge Based Systems of the IIBM Germany Scientific Center. Trans lexis aims at the theoretically and cmpirically well motivated lexical description and the management of the lexical information of LM'I in a database. It is conceived as a first step towards a reusable lexical knowledge base.

\subsection{Requirements for convenient construction and maintenance of Lexicons}

Based on our experience and existing literature, a tool for the construction and maintenance of large NLP lexicons with a complex entry structure should meet the following requirements:

- Adequate expressive power of the representation formalism: the expressive power musi be sufficient to cover the facts of lexical description.
- Methodology for the description of lexical information: eriteria and guidelines relevant for encoding should be developed and documented.

口 Orientation towards lexicographic procedure: the design of the tool should take the logical course of the lexicographic work procedure into consideration and support it during all its steps and phases. The lexicographer should be enabled to concentrate on the lexicographic description of lexical units while the tool itself automatically takes care of the remaining tasks in Jexicon development.

- Consistency and integrity checking of the lexical data: when entries are added or updated, the system should reject invalid values for particular features and check if the input leads to inconsistency of the database.

- Data independence: An extreme dependency between the structuring of lexical data stored in the database and the structure of the lexical entrics in a given application system should be avoided. In this way the lexical data will remain resistant to modifications in the NLP/MI'-systems that make use of these data.

口 Reusability/Reversability of the data (cf. Calzolari 1989, Heid 1991): lexical data should be represented in such a way that it can - apart from its transfer specific components - be reused for other MT-prototypes with the same source or target language, or with the reverse language pair (e.g. German-English and English-German). Ideally, the lexical data should be independent to such a degree that they are also rcusable for other NL.P-applications.

- Multi-user access: it should be possible for several users to work on the lexicon simultaneously.

a Help facilities: the criteria and guidelines for lexical description should be easily accessible. The availability of monolingual and bilingual dictionaries are to support the lexicographer's linguistic competence.

\section{$1.2 L M T$}

LMT, developed by Michael McCord, is in basic design a source-based transfer system in which the source analysis is done with Slot Grammar (cf. McCord 1989, 1990, forthcoming). Two main characteristics of LMT should be emphasized:

1. the lexicalism, arising from Slot Grammar source analysis; 
2. a large language-gencral $X$-to- $Y$-translation shell.

Both features facilitate the development of prototypes for new language pairs ${ }^{1}$. Versions of I.MT (in vatious stages) exist cutrently for nine lariguage pairs.

I M' I currently requires the following types of information to be specified for lexical units (I,U):

o part of specch;

c) word senses:

u morphological properties;

[) agreement features;

n the valency, i.e. the frames of optional/obligatory complenent slots;

D the specification of the fillers (NI's, subordinate clauses) for each slot;

c semantic compatibility constraints and collocations;

- characterization of multiword lexemes;

u subject area;

¿ translation relations;

n lexical transformations.

In MeCord (fortheoming), an external lexical format (IIJI) is presented which allows the representation of the above information. Intil now, however, the lexical data has been kept in sequential files and updating has been done with a text editor. Thus most of the above-mentioned requirements could not be met.

\subsection{Vocabulary Acquisition}

The hand-coding of dictionaries is a laborious and time-consuming task. Therefore a number of attempts have been made to exploit corpora and/or machine reactable dictionaries (MRIOs) for the build-up of NI,P-lexicons (cf. 3.5) ${ }^{2}$. In many cases, however, the lexical information in MRI)'s is neither complete nor sufficiently explicit for NLP/MT purposes and has to be revised by lexicographers. Irleally, the demands on a lexicographer should only be of linguistic nature. I or this reason a sophisticated tool is needed to guide and support the NI,P/MI-lexicographer in revising chitries automatically converted from machine readable sources as well as in building up new vocabulary.

\section{LOLA - architecture and components}

The lexical data base tool I.OL $\Lambda$ aims at meting the above mentioned requirements. Its design and development are based on work achicved in the I,EX-project and the COIJiX-project ${ }^{3}$. LOI $\Lambda$ makes use of automatic consistency and integrity checks as well as of the support of multi-user access provided as standard facilities by the relational
DBMS SQL/DS. Updates are made with the help of a user interface that supports the lexicographer during the cocoding process. The representation of the lexical data has been worked out to be as independent as possible of the format of a specific application lexicon, thus increasing the degree of reusability of the lexical data. In addition, a catalogue of criteria and guidelines for lexical description is being elaborated and will be integrated into the tool.

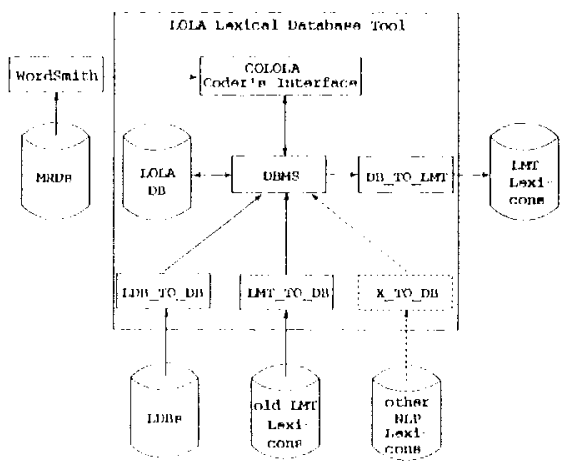

Figure I. I, OI A - architecture

The main components of the architecture of the I,OI $\Lambda$ system are the following (of. Iigure 1):

1. L,OLA-DIB: the database itself.

2. COIOOA (COder's interface to LOIA): Interface for band-coding and modification of the loxical data, stored in $I,()), A-I) B$.

3. DIS TO IMMT: program that generates I,MT lexicon entries from the lexical data stored in I. () I $\Lambda$ - IDH.

4. I,MI IO I)13: program that loads already $\mathrm{cx}$ isting $\overline{\mathrm{I}}, \mathrm{MT}$ lexicons into I,OH $\mathrm{A}-\mathrm{D}) \mathrm{B}$.

5. I,I) TO DB: program that converts data from MRT's into [.OI $\triangle-\mathrm{D}$ J].

In the following we give a brief description of these components.

\subsection{The Database}

'The database was designed in two steps: development of the conceptual sclicme and development of the database scheme.

In the conceptual design phase, the lexical objects, their properties, and their interrelations were represented in an entity-relationship diagram (cf.

1 I.M'T is the technical basis of an international project at IBM with cooperation between IBM Research, the IBM Science Centers in Heidelherg, Madrid, Paris, Ilaifa, and Cairo, and IBM Luropean Ianguage Services in Copeshagen (c. Rimon et al. 1991).

2 Cr. Byrd et al. 1987; for an overview of retated activities within the L.M'T-project, cf. Rimon ct al. 1991, pp. 14-15.

3. Cr. Barnett et al. 1986; Blumenthat et al. 1988; Storrer 1990. 
Chen 1976). Although the ER-model does not have the expressive power to cover all aspects of lexical description, especially complex constraints, it has been chosen here as a compromise between a complete lexical representation and the realization in a traditional database system.

The resulting ER-diagram for the German-Linglish lexicon is shown in Figure $2^{4}$.

The conceptual scheme is still independent of the choice of a specific DBMS and of other implementation aspects. The basic principles of the conceptual design of our database will be sketched out in the following.

Orientation towards linguistic structure, not towards the structure of the application lexicon.

The diagram reflects, in the first place, the structure of the linguistic objects, their properties and and their interrelations, and it is influenced to a smaller degree by the structure of the application lexicon. As a consequence, the data is quite resistant to structural changes in the format of the application lexicon. The abstraction from the structures of the application lexicon has a positive sidc effect with regard to the exploitation of machine readable lexical resources: on one hand, we can handle cases, in which not all information required by LMT is provided in the entries of MRD's. The information acquired can be stored as entries to be completed and revised later. On the other hand, we are free to store types of lexical information that are of relevance for NLP applications and can be acquired from MRD's or other NL.P lexicons but are not processed in a current LMT-version. We can save them in the databasc as coding aids for the lexicographers, for future prototype versions, or other NLP applications.

Analogous structure for source and target language wherever possible.

The lower part of the ER-diagram represents the German source, the upper part the English target language. For both languages, an entity of the type entry can have one or more homonyms, each of which can have one or more senses. The senses themselves can open one or more sense-specific slots (one-to-many rclations). $\Lambda$ sense-specific slot can be filled by several types of fillers and the same type of filler can fill several sense-specific slots (manyto-many relation). The basic types of entities and relations, which are the same for all languages, are described by their charactcristic features represented as attributes. The number of attributes as well as their values may differ according to languagespecific peculiarities ${ }^{5}$.

Many-to-many relations between the lexical objects of both languages.

We represent the relation of lexical equivalence between source and target senses. as a many-to-many relation (one source sense can have multiple target equivalents and vice versa). This breaks with the traditional hicrarchical entry structure of bilingual dictionaries (Calzolari et al. 1990), but it avoids redundant description and storage of one target sense that is lexically equivalent to different source senses. Another relation holds for the sense-specific slots of two senses that are regarded as lexically equivalent. We decided to establish this relation between slots and not between slot frames. This way we can elegantly describe lexically equivalent senses with non-corresponding slotframes ${ }^{6}$. In this way the relations between the two languages may be used to a great extent bidirectionally for the $\mathrm{XY}$ - as well as for the $\mathrm{YX}$-language pair.

The conceptual scheme captured in the ER-diagram was then mapped into a database scheme and implemented in the relational DBMS SQL/DS. We chose a relational DBMS, because - for the maintenance of the large LMT-GE lexicon (about 50,000 entries) - we were in need of a stable DBMS which supports multi-user access, has facilities for automatic checking of consistency and integrity of the lexical data and allows for the specification of multiple user-specific views on the data. To avoid redundancy and update anomalies we tried to normalize our relations as far it was useful with respcet to our approach. In total, 32 tables are implemented: 25 tables describe lexical objects and rclations by means of attributes with associated values, 7 tables serve to store "knowledge about the lexical knowledge", e.g. the admitted values for attributes such as semantic type, filler-type, slot-type for both languages.

\subsection{COLOLA: the user interface to LOLA}

COLOLA is the user interface to LOLA-DB that looks up the lexical data of a given search word and displays it on sequentially connected menus. The design of the menus as well as their sequential order was guided by the manner in which lexicographers describe lexical entries. The following operations can be performed:

4 The boxes represent types of entities, the diamonds represent types of relations between entities, the ellipses represent attributes which characterize types of entities or relations. The labels of the connection lines indicate whether the relation in question is a one-to-one, one-to-many, many-to-one or many-to-many relation.

The ER-diagram is a simplified version of the actual conceptual model. For the purpose of this paper, several entity types, attributes, and relations have been lef out.

5 E.g.: in German a preposition like "auf" can govern either an accusative NP ("warten auf") or a dative NP ("lasten auf") depending on the verb that takes the prepositional phrase with the respective preposition as a complement. Therefore "case" is a feature, relevant for the description of German slot fillers filling a prepositional complement slot.

- E.g. cases like "like" and "gefallen" where the subject of the English verb corresponds to the dative object of the German verb; or cases like "geigen" and "play the violin" where the English direct object filler "the violin" is incor. porated in the semantics of the German verb "geigen". 


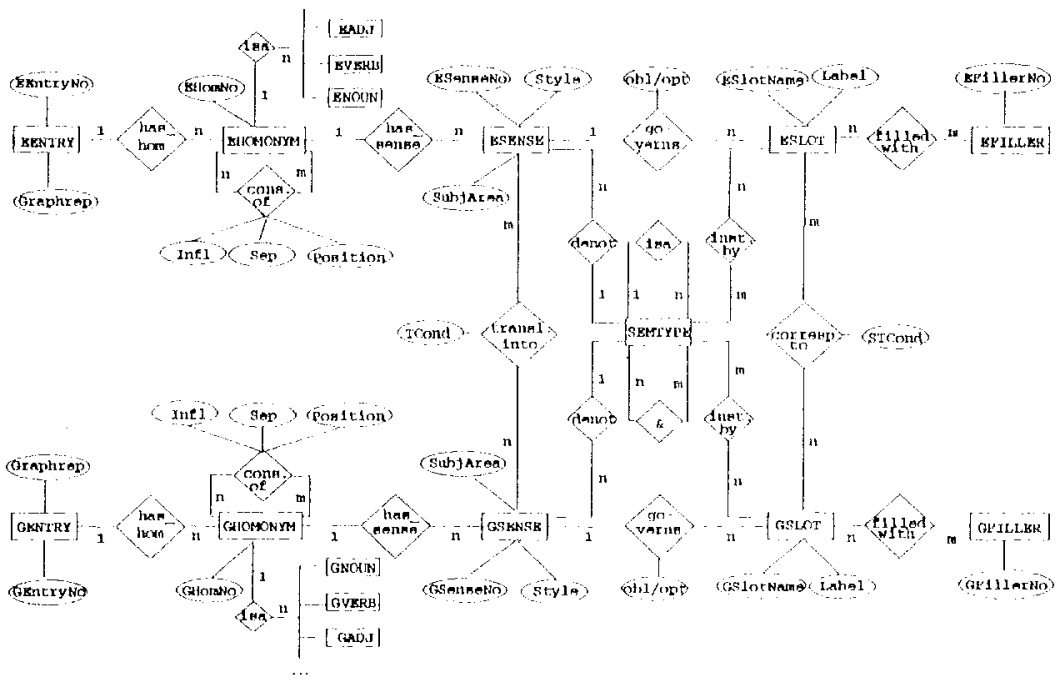

Figure 2. Ėntity Relationship Diagram for German-Linglish

․ addition of new source or target entries

a deletion of existing entries

- change of existing or addition of new features to existing entries

ㄱ deletion of features from existing entries

口 assignment of new or deletion of existing translation equivalents for a given source sense a update, insertion or deletion of transfer information for each pair of translation equivalents.

For each part of speech, a specific sequence of menus is defined. There are monus for homonyms and senses of source and target entries; the "linking" of the source senses and target senses regarded to be lexically equivalent is done via transfer menus. 'I his allows lexicographers to specialize on specific parts of speech or on specific features which can be locally updated.

COLOIA controls multi-user access to the LOIA-DB so that several lexicographers can update the lexical database simultaneously. The logical unit of work is the source or target homonym: when a lexicographer requests to update a homonym, this homonym, together with its senses, is locked for other users.

If a new entry contains blanks, a multiword menu is called where the multiword is split up into its components. For each component, the following lexical information is gathered: the part of speech, whether the word may inflect within the multiword, and whether a phrase can be inserted between one multiword component and the previous one without doing away with idiomaticity.
If new homonyms or senses are inserted on the multiword menu as well as on other menus, default values for features are displityed. 'They can either be accepted or rejected and overwritten by the lexicographer ${ }^{7}$. The assumptions on default values for atributes of lexical information may differ according to different grammars and systems. We therefore decided to store the complete lexical information and use default vilues as proposals in the user interface. With this approach we allow for two advantages: on one hand, the data in the database can be used for different applications having distinct theory specific assumptions on defaults. On the other hand, the user of COI,OL $A$ can benefit from the economic advantages of default assumptions.

COIOLA does extensive consistency checking of the values entered by the lexicographers. Illegal values are rejected and wanning messages are displayed in situations where errors might casily occur. $\Lambda \mathrm{l}-$ though much of the consistency checking is supported by the database management system, some extensions were necessary.

liurther support for the lexicographers is provided by an interface to the WordSmith on-line dictionary system (cf. Byrd/Neff 1987). Several machine readable dictionaries are available e.g. Collins Germanlinglish, Hanglish-German, Longman's Ijictionary of Contemporary Jinglish, and Webster 7 th Collcgiate dictionary. The lexicographer can look up entries in these dictionarics during the encoding process. I urthermore, help menus are provided in which the valid values for specific features can be looked up.

7. Default values are provided, for instance, for slot fillers. German direct object slots get an accusative noun phrase as the default filler. 'I'he lexicographers may accept this, add other fillers or write over it with another filler. 


\subsection{DB TO LMT}

$\Lambda$ conversion program IDB TO LMT has been developed which extracts lexical information stored in the relations of $I, O 1, \triangle-D B$ and converts it into I,MT-format. DB 'IO IMY consists of two components:

u a database extractor and

口 a conversion program

The database extractor sclects the source cntries and the corresponding target cntries and stores them in database format. This format can be regarded as an intermediate represcntation between database scheme and IMI'-format. It consists of a set of Prolog predicates which correspond to the relations of the database schene. There are, for instance, entry, homonym, sense, and slot predicates which correspond to the entry, homonym, sense, and slot relations in the database. The conversion program finally converts the database format into the LMT-format. It has to be adapted according to the changes or extensions of the I.MT-fortnat.

\section{4. $L M T T O D B$}

Before and during LOL $\Lambda$ design and development, I,MT lexicons in ELFi were already created and updated in files. Since these lexicons still need updating and since this is much better supported by I,OLA, a conversion program LMT TO DB was needed which converts $1 \mathrm{BL}{ }^{+}$entries of lexicon files into the database format and loads them into I,OI A-DB. I MT TO DB consists of threc components:

a the lexicon compiler of LMI',

$\square$ a conversion component, and

u a database loader.

'The lexicon compiler is the component of the $1 \mathrm{M} T$ system which converts the liI, $\mathbf{f}^{i}$ into the internal I,M'l' format ${ }^{8}$, In the internal format all abbreviation conventions and default assumptions are atready interpreted and expanded accordingly so that the complete lexical information is represented explicitly. The conversion component then converts the internal I,MT-format to database format. 'The database loader generates the SQL-statements and updates the database. It has to check first whether the homonym or sense to be inserted is identical with an homonym or sense stored in the database. If all the features of two homonyms or senses can be unified, they are regarded to be identical and the already existing entry is merged with the converted entry. In all other cases the homonym or sense is inserted into the database and merging has to be done by the lexicographers with COLOLA.

\section{5. $\mathrm{LDB}_{-} T \mathrm{O}_{-} \mathrm{DB}$}

To supplement the lexical coverage of the LM'I system, a dictionary access module has been developed which allows rcal-time access (cf. Neff/McCord 1990) to Collins bilingual dictionaries available as lexical data bases (I,DHs)' "The module includes a language pair independent shell component COIIXY and language-specific components and converts the lexical data of the LID into the IMI'-format. I,DB_ IO_DI is based on these programs. It consists of

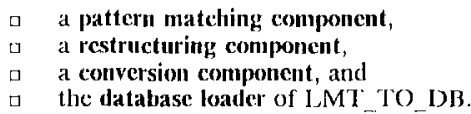

With the pattern matching component, those features (sub-trees) that are to be converted are selected from the dictionary entries. In printed dictionaries, features common to more than one sub-tree are often factored out in order to save space. With the restructuring component, those features can be moved to the sub-trees they logically belong to. The conversion eomponent converts the restructured dictionary entry to database format. The database loader of LMi $\mathrm{TO}$ DB merges the entry with a possibly atready existing one in I.OI A-DB and generates the SQI-statements to update the database. The converted entries can be revised by the lexicographers with COI.OI A.

\section{Reusability of the LOLA system}

\subsection{Reusability of the tool components}

The first I OI A prototype was developed to support lexicon development for the language pair German-linglish. In the meantime, work has been started to make the tool usable for lexicon development of the Inglish-Danish and Inglish-Spanish I.M'T systems. As a positive result of the design principles described in section 3.1., the database scheme had to be modified only slightly with regard to prototype-specific differences ${ }^{10}$. The values for language-specific attributes such as types of slots and fillers will be defined for the "new" languages Spanish and Danish and will be stored in the database. "They can then be used for consistency checking (only defined values can be updated in the database). In COI.OLA we had to take into account the homonym level on the target side, where

* In the morpho-texical processing and compiling phase, L1.J entries are converted into an internal format (cf. McCord (forthcoming): sect. 2) which represents the initial source and transfer analysis of an individual input word string.

9 An L.DI provides a tree representation of the hierarchical structure of the dictionary entries. The nodes of the tree are labeled with attributes having specific values for each individual entry. The L.DB can be queried with the specialized query language L.QI. (cf. Neff/Byrd/Rizk 1988).

10 Fnglish-Danish and English-Spanish use lexicon driven morphology for the Larget languages Spanish (cf. Rimon et at. 1991) and Danish, whereas German-English uses a rule-based target morphology for Inglish (cl. McCord/Wolff 1988 ). 
the features of Spanish and Danish morplology have to be specified. The programs that convert the database entries into the format of the application lexicons and vice versa (I)B_TO_LMT' and IMI TO DB) need generalization in order to achicve an abstraction from prototype-specific features of I.M'I'.

\subsection{Reusability of the lexical data}

In order to meet the requirement of data independence, the representation of lexical entrics in the database is highly independent of that in the application lexicon. In the database, the description of linguistic entities and their interrclations is given in at set of tables where specific values are stored for the characteristic attributes of each individual entity. On these tables, different views can be defined for different types of users. Different programs (like DB 'TO_IMT') can extract exactly the atribute values needed for their respective application and convert them into each given format. 'I his way, from one and the same data base several lexicons can be gencrated, in which the same linguistic world is structured differently or represented in a completely different way. The possibilitics of reusability are naturally defined and limited by the number of the registered types of lexical information in the original data base. $\Lambda$ s far as the $101 . \Lambda \mathrm{da}$ tabase is concerned, the very detailed description of slot frames as well as the information about multiwords and the propertics of their components may be reused for other NL.P applications with one of the languages involved, The reusability of the transfer information (specified in the transfer relations between the languages of a given language pair) for other MT systems depends highly on the respective M'T approach. $\Lambda s$ to the question of reusabilty of the data in the I,MT system "family", three different cases have to be distinguished:

1. lexical-data description given for a source language $X$ is reused for another language pair having $X$ as source language,

2. Exical-data description given for a source languagc $X$ is reused for another language pair having $X$ as target language,

3. lexical-data description given for a target language $Y$ is reused for another language pair having $Y$ as source language.

In the first two cases, reusability of the lexical data of language $X$ is very high. In the third case, the description of $Y$ as source language may have to be more detailed in order to achieve an adequate syntactic analysis ${ }^{1 t}$. New attributes or even ncw types of entities or relationships may be needed and the database scheme will have to be enhanced accordingly.

\section{Outlook}

Our long-term goal is a multilingual database, in which the lexical knowledge for each language involved in the I.MT project is represented only once.

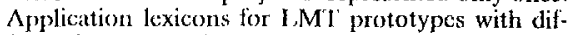
ferent language pairs are generated by extracting the required information from the database and by converting it into the respective IMT'format. Furthermore, the tool is to be extended in such a way that it is not restricted to the construction of MT lexicons, but cain also be used as a terminology workbench and thus support the construction and naintenance of terminology. $\Lambda_{n}$ integrated $M^{\prime} T$ and terminology database would have the advantage that the lexical knowledge cncoded by terminologists and translators can be used by the translation system as well. for refinement and completion of the description of the German language, it is plamed to integrate further information from available German NLP lexicons into the IOI $\Lambda-D B$. $\Lambda$ basic problem conecrning this undertaking will be to identify and to match the basic categories "entrics", "homonyms", "senses", which are defined in various lexical resources according to different eriteria, only some of which being transparent. With this effort, we hope to gain further knowledge on the limits and possibilities concerning the reusability of lexical data.

\section{References}

Barnett, B., II. L.ehmann, M. Koeppritz (1986): " $\Lambda$ Word Database for Natural I anguage Processing", Proceedings $1 /$ th International Conference on Computational linguistics COLING86 August 25th to 29th, 1986, Bonn, Federal Republic: of Germany, pp. 435-440.

Bhumenthal et a1. (1988); "Was ist eigentlich ein Verweis? - Konzeptuclle Datenenodellicrung als Voraussetzung computergestützter Verwcisbehandlung." , Ilarras,G.(ed.): Das Wörterbuch. Artikel und Verweisstrukturen. Düsseldorf 1988.

Byrd, R., N. Calzolatri, M. Chodorow, J. Klavans, M. S. Neff, O. Rizk(1987): "Tools and Methods for Computational lexicography", Computational Linguistics, 13, 3-4.

Byrd, R. J., M. S. Neff (1987): WordSmith User's Guide, Research Report, IBM Rescarch Division, Yorktown I leights, NY 10598.

Chen, Peter P.-S. (1976): "'The lintity-Relationship Model- Towards a Unified View of Data" , $A C M$ I'ransactions on Database Systems 1, pp. 9-36.

Calzolari, N. (1989): "The Development of I-aige Mono- and Bilingual I exical Databases", Contribution to the IBM Europe Institute "Computer based

I Jig. in a source-based translation system like I.MY', the information on whether a target slot is obligatory is not direcly encoded in the I.MT transfer-lexicon; the system controls target slot assignment by the preserice of a corresponding source stot in a given input sentence and the mapping relation specilied within the transfer lexicon entry. On the source side, however, the feature of slot obligatoriness is used for purposes of analysis disambiguation. 
Translation of Natural Language", (GarmischPartenkirchen.

Calzolari et al. (1990): "Computational Model of the Dictionary I'ntry - Preliminary Repott", Project AQUILEX, Pisa.

Heid, U. (1991): A short report on the EUROTRA-7 Study, Univ. of Stuttgart 1991.

MeCord, M. C. (1989) "A New Version of the Machine 'Translation System LM'T", I.terary and Linguistic Computing, 4, pp. 218-229.

McCord, M. C. (1990): "Slot Grammar: A System for Simpler Construction of Practical Natural $I_{\text {ritn- }}$ guage (irammars", In R. Studer (ed.), Natural Language and Logic: Iniernational Scientific Symposium, Lecture Notes in Computer Science, Springer Verlag, Berlin, pp. 118-145.

McCord, M. C. (forthcoming): "The Slot Grammar System", In J. Wedekind and Ch. Rohrer (cd.), Unification in Grammar, to appear in MIT Press.

McCord, M. C., Wolff, S. (1988): The Lexicon and Morphology for LMT, a Prolog-based MT system,
Rescarch Report RC 13403, IBM Research Division, Yorktown Heights, NY 10598.

Neff/Byrd/Rizk (1988) M. S. Neff, R. J. Byrd, O. A. Rizk: "Creating and Querying Flierarchical I sxical Data Bases", Proceedings of the 2nd ACL Conference on Applied NLP, 1988.

Neff, M. S., M. C. McCord (1990): "Acquiring Lexical Data From Machine-readable Dictionary Resources for Machine Translation", Proceedings of the 3rd Int. Conf. on Theoretical and Melhodological Issues in Machine Translation of Natural Languages, pp. 87-92. Linguistics Research Center, Univ. of T'exas, Austin.

Storrer, A. (1990); "Uberlegungen zur Repräsentation der Verbsyntax in einer multifunktional-polytheoretischen lexikalischen Datenbank", Schaeder,B./Rieger,B. (ed.): Jexikon und Lexikographie: maschinell - maschinell gestützt. Grundlagen - Entwicklungen - Produkte, Hildesheim, pp. 120-133.

Rimon, M., McCord, M. C., Schwall, U., Martínez, P. (1991): "Advances in Machine 'Iranslation Rescarch in IBM," Proceedings of MT Summit III, pp. 11-18, Washington D.C. 